\title{
CONDUTIVIDADE ELÉTRICA DA SOLUÇÃO NUTRITIVA E PRODUÇÃO DE ALFACE EM HIDROPONIA
} \author{
Rumy Goto ${ }^{3 *}$ \\ ${ }^{1}$ Estagiárias do Depto. de Produção Vegetal-Horticultura, FCA/UNESP. \\ ${ }_{2}^{2}$ Pós-graduandos do Depto. de Produção Vegetal-Horticultura, FCA/UNESP. \\ ${ }^{3}$ Depto. de Produção Vegetal, FCA/UNESP, C.P. 237 - CEP: 18603-970 - Botucatu, SP. \\ *Autor correspondente <rumy@fca.unesp.br>
}

Paulo César Costa²; Evanessa B. Didone”; Thaís M. Sesso; Kathia Alexandra Lara Cañizares²;

RESUMO: A variação da condutividade elétrica da solução nutritiva altera a absorção de água e nutrientes pelas plantas, interferindo no metabolismo e, consequentemente, na produção das mesmas. Este trabalho, conduzido no período de agosto a outubro de 1999, avaliou o efeito da condutividade elétrica (CE) sobre a produção e desenvolvimento de alface americana de cabeça crespa, cultivar Ryder em hidroponia. As tendências de variação da condutividade elétrica do meio nutritivo, durante o desenvolvimento da alface, foram também avaliadas. Foi utilizado o delineamento inteiramente casualizado com seis repetições e três tratamentos, constituídos pelas condutividade elétricas: $\mathrm{CE}_{1}=1,46, \mathrm{CE}_{2}=2,46$ e CE$_{3}=4,21( \pm 0,24) \mathrm{mS} \mathrm{cm}^{-1}$. O diâmetro e altura da cabeça e peso seco da raiz não foram influenciados pelas condutividades elétricas da solução nutritiva. Os maiores pesos do material fresco e seco foram conseguidos com CE 2,46 $( \pm 0,24)$ $\mathrm{mS} \mathrm{cm}^{-1}$. Todos os tratamentos apresentaram a tendência de diminuição da condutividade elétrica, durante 0 cultivo da alface verificando-se que a condutividade elétrica teve influência sobre as características de produção da alface americana.

Palavras-chave: Lactuca sativa, solução nutritiva, produção, cultivo sem solo

\section{ELECTRICAL CONDUCTIVITY OF NUTRIENT SOLUTION AND HIDROPONIC CRISP HEAD LETTUCE YIELD}

\begin{abstract}
The levels of electrical conductivity (EC) can alter water and nutrient uptake by plants, influencing their metabolism and yield. This experiment was carried out to verify the effects of EC on the yield and the development of the crisp head lettuce cv. Ryder in hidroponics. The tendency of the electrical conductivity changes of the nutrient solution during the development of the lettuce was also evaluated. A completely randomized design was used, with six replications and three treataments: $\mathrm{EC}_{1}=1.46, \mathrm{EC}_{2}=2.46$ and $\mathrm{EC}_{3}=$ $4.21( \pm 0.24) \mathrm{mS} \mathrm{cm}^{-1}$. Lettuce head diameter and height, and root dry weight were not influenced by EC. The largest fresh and dry weights of heads were obtained for $2.46( \pm 0.24) \mathrm{mS} \mathrm{cm}^{-1}$. All treatments had the same tendency of reducing EC during lettuce growth.

Key words: Lactuca sativa, nutritive solution, yield, soilless culture
\end{abstract}

\section{INTRODUÇÃO}

O cultivo da alface em sistemas hidropônicos, principalmente o NFT, já é bastante difundido no Brasil, principalmente pelo fácil manejo que a cultura permite, aliado ao seu ciclo curto (Gualberto et al, 1999). O cultivo dessa hortaliça, em hidroponia, pode ser adequado para que se obtenha maiores produções, controlando-se as condições do meio nutritivo (Jensen \& Collins, 1985). Essa possibilidade de controle é uma das principais vantagens conferidas à hidroponia, dadas a rapidez e a facilidade com que isso pode ser feito.

Dentre as muitas propriedades apresentadas por uma solução nutritiva, cita-se a condutividade elétrica. Existe muita controvérsia com relação ao melhor valor de condutividade elétrica a ser adotado para o cultivo da alface em hidroponia. Acredita-se também que esses valores devem variar de acordo com a cultivar adotada, bem como com as condições climáticas. Os valores de condutividade elétrica são proporcionais à concentração dos vários íons em solução, e da mesma forma ao potencial osmótico da mesma. De acordo com Bresler \& Hoffman (1986) a absorção de água pelas plantas, através do sistema radicular, é influenciada pelo potencial osmótico do meio nutritivo. Huet (1994) afirma que a condutividade da solução nutritiva não influencia somente a absorção de água, mas também a absorção de nutrientes, estando ambas intimamente ligadas.

As mudanças na absorção de água e nutrientes, proporcionadas pela variação da condutividade do meio nutritivo, levam a alterações da fisiologia das plantas (Beltrão et al, 1997). Essas alterações relacionam-se, dentre outros fatores, à abertura estomática e ao aumento ou diminuição da área foliar, estando esses fatores intimamente ligados com a eficiência fotossintética, e consequentemente, com a produção de material seco pelas plantas. 
Baseado no exposto, este trabalho objetivou estudar o efeito da condutividade elétrica da solução nutritiva sobre a produção de alface em hidroponia, acompanhando a variação da condutividade da solução durante o ciclo da cultura.

\section{MATERIAL E MÉTODOS}

O experimento foi conduzido nas dependências do Departamento de Produção Vegetal, Área de Horticultura da FCA - UNESP - Botucatu - SP. Sementes de alface americana, cultivar Ryder, foram colocadas para germinar em células de espuma fenólica com dimensões de $2 \times 2 \times 2 \mathrm{~cm}$. As sementes foram colocadas em orifícios com cerca de $0,5 \mathrm{~cm}$ de diâmetro e $1,8 \mathrm{~cm}$ de profundidade feitos em cada célula da espuma fenólica. Após a colocação das sementes, as placas de espuma fenólica foram umidecidas diariamente com água pura até a acorrência da germinação.

Após a germinação as plântulas foram transferidas para um berçário, quando passaram a receber a solução com a seguinte composição, em $\mathrm{mg} \mathrm{L}^{-1}: \mathrm{N}=90$; $\mathrm{K}=84 ; \mathrm{Ca}=110 ; \mathrm{P}=20 ; \mathrm{Mg}=15 ; \mathrm{S}=22 ; \mathrm{B}=0,25$; $\mathrm{Mn}=0,25 ; \mathrm{Cu}=0,025 ; \mathrm{Mo}=0,01 ; \mathrm{Fe}=2,5 ; \mathrm{Zn}=0,05$.

Aos 30 dias após a semeadura, mudas com seis folhas definitivas foram transferidas, no dia 04/09/1999, para caixas de poliestireno expandido com 17 litros de capacidade. As mudas foram presas na altura do colo, com auxílio de espuma sintética, transpassadas por orifícios com cerca de $3 \mathrm{~cm}$ de diâmetro, feitos no centro das tampas das caixas. As caixas foram preenchidas com 15 litros de solução nutritiva. Nas tampas, além do orifício central, no qual foram colocadas as plantas, haviam orifícios laterais, para garantir a troca de gases entre 0 interior e o exterior das caixas, e permitir as leituras de $\mathrm{pH}$ e condutividade elétrica das soluções. As caixas foram dispostas uma ao lado da outra, de modo que o espaçamento apresentado era de 40×25 cm entre plantas.

$O$ delineamento experimental, inteiramente casualizado, consistiu de três tratamentos, com seis repetições, com uma planta por parcela. Os tratamentos corresponderam a três diferentes valores de condutividade elétrica, sendo eles: $C E_{1}=1,46 ; \mathrm{CE}_{2}=2,46$; $\mathrm{CE}_{3}=4,21( \pm 0,24) \mathrm{mS} \mathrm{cm}^{-1}$.

As soluções nutritivas utilizadas foram adaptações feitas a partir do balanço de solução nutritiva proposto por Sarruge (1975). A solução nutritiva que correspondeu a $\mathrm{CE}_{3}=4,21( \pm 0,24) \mathrm{mS} \mathrm{cm}^{-1}$ possuía a seguinte composição de macronutrientes, em $\mathrm{mg} \mathrm{L}^{-1}: \mathrm{N}=$ $360 ; \mathrm{K}=336 ; \mathrm{Ca}=440 ; \mathrm{P}=80 ; \mathrm{Mg}=60 ; \mathrm{S}=88$. A solução nutritiva que correspondeu a $\mathrm{CE}_{2}=2,46( \pm 0,24)$ $\mathrm{mS} \mathrm{cm}^{-1}$ possuía a seguinte composição de macronutrientes, em mg L ${ }^{-1}: \mathrm{N}=180 ; \mathrm{K}=168 ; \mathrm{Ca}=220$; $P=40 ; M g=30 ; S=44$. A solução nutritiva que correspondeu a $\mathrm{CE}_{1}=1,46( \pm 0,24) \mathrm{mS} \mathrm{cm}^{-1}$ possuía a seguinte composição de macronutrientes, em $\mathrm{mg} \mathrm{L}^{-1}: \mathrm{N}=$ $90 ; \mathrm{K}=84 ; \mathrm{Ca}=110 ; \mathrm{P}=20 ; \mathrm{Mg}=15 ; \mathrm{S}=22$.
A composição de micronutrientes, utilizada nas três soluções, foi a seguinte, em $\mathrm{mg} \mathrm{L}^{-1}: \mathrm{Fe}=5 ; \mathrm{B}=0,5$; $M n=0,5 ; Z n=0,1 ; C u=0,05 ; M o=0,02$.

A água evapotranspirada era reposta, semanalmente, com adição de água pura, sem reposição de nutrientes. Os valores de condutividade elétrica e $\mathrm{pH}$ das soluções nutritivas eram tomados semanalmente. Não foram necessárias correções do $\mathrm{pH}$ das soluções nutritivas, pois este variou entre 5,5 e 6,5, durante todo o experimento, tendo-se essa faixa como ótima.

Com os valores da conduvidade elétrica da solução, registrados durante o ciclo da cultura, foram ajustadas equações de regressão. As características avaliadas após a colheita foram: diâmetro, altura e peso da matéria fresca e seca da cabeça; e peso da matéria seca da raiz. Os resultados obtidos foram submetidos à análise de variância e as médias foram comparadas pelo teste de Tukey a $5 \%$.

\section{RESULTADOS E DISCUSSÃO}

$\mathrm{Na}$ solução nutritiva com condutividade inicial $1,46( \pm 0,24) \mathrm{mS} \mathrm{cm}^{-1}$, observou-se redução constante da condutividade elérica, chegando a $0,6 \mathrm{mS} \mathrm{cm}^{-1} \mathrm{na}$ última semana, marcando um decréscimo linear da $C E$ com $R^{2}=0,95$ (Figura 1). A redução da condutividade elétrica foi devida à absorção dos nutrientes pelas plantas, pois os nutrientes não foram repostos durante o ciclo da cultura, somente a água evapotranspirada.

Nas soluções nutritivas com condutividades iniciais de 2,46 $\left(\mathrm{CE}_{2}\right)$ e 4,21 $\left(\mathrm{CE}_{3}\right) \pm 0,24 \mathrm{mScm}^{-1}$, os valores de condutividade apresentaram uma tendência polinomial. A curva de $C E_{2}$ apresentou $R^{2}=0,92$ sendo estatisticamente significativo. A curva de $\mathrm{CE}_{3}$ apresentou $R^{2}=0,65$ sendo estatisticamente não significativa (Figura 1).

As plantas mantiveram mesma magnitude de absorção, baseando-se na variação da condutividade. Porém, as condutividades iniciais consideradas influenciaram de forma diferente o crescimento das plantas, sendo que as plantas (parte aérea e raiz) apresentaram os seguintes pesos de material seco em função das respectivas condutividades: $\mathrm{CE}_{1}=35,32 \mathrm{~g}$; $\mathrm{CE}_{2}=46,01 \mathrm{~g} ; \mathrm{CE}_{3}=30,00 \mathrm{~g}$.

O diâmetro e altura da cabeça da alface e peso da matéria seca das raízes não apresentaram diferenças significativas, levando a inferir que a condutividade elétrica não teve efeito sobre essas características (TABELA 1).

A produção de material fresco e seco da cabeça foram favorecidos quando a condutividade da solução nutritiva estava em torno de 2,46 $\pm 0,24 \mathrm{mS} \mathrm{cm}^{-1}$, concordando com o que constatou Economakis (1990) com alface de cabeça, cv. Bellona. A menor produção de material seco deu-se em função da maior condutividade, concordando com o que foi exposto por Beltrão et al (1997), em que os autores afirmam que 
TABELA 1 - Diâmetro, altura, matéria fresca e seca da cabeça e matéria seca da raiz de plantas de alface cultivadas em solução nutritiva em três condutividades elétricas. UNESP, Botucatu, 1999.

\begin{tabular}{|c|c|c|c|c|c|}
\hline \multirow[b]{2}{*}{ Tratamento } & \multirow[b]{2}{*}{ Diâmetro cabeça } & \multirow[b]{2}{*}{ Altura cabeça } & \multicolumn{3}{|c|}{ Peso } \\
\hline & & & $\begin{array}{c}\text { maté ria fresca } \\
\text { cabeça }\end{array}$ & $\begin{array}{c}\text { matéria seca } \\
\text { cabeça }\end{array}$ & $\begin{array}{c}\text { matéria seca } \\
\text { raiz }\end{array}$ \\
\hline & 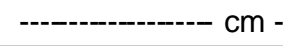 & 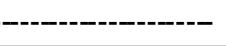 & 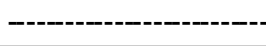 & ---- g ----------- & 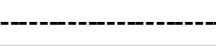 \\
\hline CE1: $1,46 \pm 0,24$ & $15,32 \mathrm{a}$ & $12,83 \mathrm{a}$ & $451,90 \mathrm{~b}$ & $16,23 b$ & $3,22 \mathrm{a}$ \\
\hline CE2: $2,46 \pm 0,24$ & $16,78 \mathrm{a}$ & $13,67 \mathrm{a}$ & $604,98 \mathrm{a}$ & $24,50 \mathrm{a}$ & $3,28 \mathrm{a}$ \\
\hline CE3: $4,21 \pm 0,24$ & $12,78 \mathrm{a}$ & $11,67 \mathrm{a}$ & $195,95 \mathrm{c}$ & $8,12 \mathrm{c}$ & 3,38 a \\
\hline $\mathrm{CV}(\%)$ & 22,97 & 15,20 & 20,07 & 24,39 & 25,10 \\
\hline
\end{tabular}

Médias seguidas por letras iguais na mesma coluna não diferem pelo teste de Tukey a 5 \%.

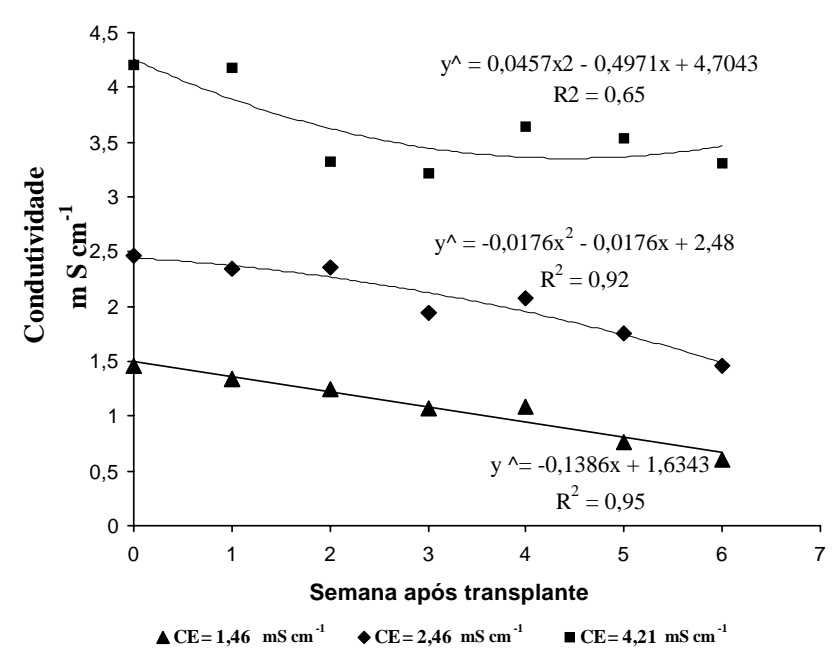

aumentos na condutividade levam a diminuição da produção de material seco, bem como da produtividade.

Segundo Huett (1994), plantas de alface (cv. Coolguard) cultivadas em baixa condutividade elétrica $\left(0,4 \mathrm{mS} \mathrm{cm}^{-1}\right)$, apresentaram deficiências de nitrogênio e potássio, e altos teores de cálcio em folhas novas, sendo que as deficiências diminuiram com o aumento da condutividade elétrica da solução nutritiva. Embora não tenham sido apresentados dados relativos a análises químicas de folhas, a baixa absorção de nitrogênio e potássio pode ter sido a causa da menor produção de material seco e fresco na menor condutividade.

\section{CONCLUSÕES}

A condutividade elétrica da solução nutritiva influenciou o peso do material fresco e seco da cabeça de alface, sendo o maior valor obtido com a CE de 2,46 $\pm 0,24 \mathrm{mS} \mathrm{cm}^{-1}$. Essa condutividade foi a mais indicada para o cultivo de alface americana, cultivar Ryder, em hidroponia, nas condições estudadas.

\section{REFERÊNCIAS BIBLIOGRÁFICAS}

BELTRÃO, J.; TRINDADE, D.; CORREIA, P.J. Lettuce yield response to salinity of. sprinkle irrigation water. Acta Horticulturae, v.449, p.623-627, 1997.

BRESLER, E.; HOFFMAN, G.J. Irrigation menagement for soil salinity control: theories and tests. Soil Science Society of America Journal, v.50, p.1552-1560, 1986.

ECONOMAKIS, C.D. Effect of solution conductivity on growth and yield of lettuce in nutrient film culture. Acta Horticulturae, v.287, p.309-316, 1990.

GUALBERTO, R.; RESENDE, F.V.; BRAZ, L.T.. Competição de cultivares de alface sob cultivo hidropônico "NFT" em três diferentes espaçamentos. Horticultura Brasileira, v.17, p.155-158, 1999.

HUETT, D.O. Growth, nutrient uptake and tipburn severity of hidroponic lettuce in response to electrical conductivity and $\mathrm{K}: \mathrm{Ca}$ ratio in solution. Australian Journal of Agricultural Research, v.45, p.251-267, 1994.

JENSEN, M.H.; COLLINS, W.L. Hydroponic vegetable production. Horticultural Reviews, v.7, p.484-555, 1985.

SARRUGE, J.R. Soluções nutritivas. Summa Phytopatologica, v.1, p.231-233, 1975.

$\overline{\text { Recebido em } 02.05 .00}$ 